\title{
Characterization of X3 Silicon Detectors for the ELISSA Array at ELI-NP
}

\author{
S. Chesnevskaya ${ }^{1, \star}$, D. L. Balabanski ${ }^{1}$, D. Choudhury ${ }^{1}$, M. La Cognata $^{2}$, P. Constantin ${ }^{1}$, D. M. \\ Filipescu $^{1}$, D. G. Ghita ${ }^{1}$, G. L. Guardo ${ }^{1,2}$, D. Lattuada $^{1,2}$, C. Matei ${ }^{1}$, A. Rotaru ${ }^{1}$, C. Spitaleri. ${ }^{2}$, A. \\ State $^{1}$, and $Y . \mathrm{Xu}^{1}$ \\ ${ }^{1}$ ELI-NP/IFIN-HH, 30 Reactorului Street, 077125, Magurele, Romania \\ ${ }^{2}$ INFN, Laboratori Nazionali del Sud, Via Santa Sofia 62, 95123 Catania, Italy
}

\begin{abstract}
Position-sensitive silicon strip detectors represent one of the best solutions for the detection of charged particles as they provide good energy and position resolution over a large range of energies. A silicon array coupled with the gamma beams at the ELINP facility would allow measuring photodissociation reactions of interest for Big Bang Nucleosynthesis and on heavy nuclei intervening in the p-process. Forty X3 detectors for our ELISSA (ELI-NP Silicon Strip Detectors Array) project have been recently purchased and tested. We investigated several specifications, such as leakage currents, depletion voltage, and detector stability under vacuum. The energy and position resolution, and ballistic deficit were measured and analyzed. This paper presents the main results of our extensive testing. The measured energy resolution for the $\mathrm{X} 3$ detectors is better than results published for similar arrays (ANASEN or ORRUBA).
\end{abstract}

\section{Introduction}

A $4 \pi$ silicon-strip detector array with removable solid target, ELISSA, has been designed in collaboration with INFN-LNS, Catania [1-3]. The ELISSA array consisting of three rings of twelve single-sided X3 detectors, and two end-caps made up of four double-sided QQQ3 detectors from Micron Semiconductor [4] is under construction at the Extreme Light Infrastructure-Nuclear Physics (ELI-NP) facility in Magurele, Romania. This type of array has already been successfully applied for nuclear astrophysics studies, e g. ORRUBA (Oak Ridge Rutgers University Barrel Array) or ANASEN (Array for Nuclear Astrophysics Studies with Exotic Nuclei) [5, 6].

\section{X3 detector testing: results and discussion}

The X3-type detectors are $1000 \mu \mathrm{m}$ thick and have an active area of $7.5 \mathrm{~cm} \times 4 \mathrm{~cm}$. A resistive layer of doped silicon divided into four 1-cm wide strips is the front junction side. The rear ohmic side is non-resistive. The experimental test setup consists of the X3 detector and $\alpha$-source assembly located inside a large cubicle vacuum chamber, also designed to accommodate the signal read-out chain. The $\mathrm{X} 3$ detector was attached to a holder fixed to a heavy aluminum plate. A standard two-peak open

^e-mail: svetlana.chesnevskaya@eli-np.ro 
$\alpha$-source, ${ }^{241} \mathrm{Am} /{ }^{239} \mathrm{Pu}$, with an activity of $1.035 \mathrm{kBq}$, was placed in a cylindrical canister closed with a lid of selectable collimator hole. The hole diameter of $2 \mathrm{~mm}$ and the distance between the $\alpha$-source and the surface of the detector of $25 \mathrm{~cm}$ are chosen so as to illuminate two strips simultaneously. The $\alpha$-source can be moved vertically and horizontally with respect to the fixed X3 detector using two stepper motors controlled via LabView Software. The motors are driving threaded rods with a thread pitch of $1 \mathrm{~mm}$.

The energy resolution was measured for all X3 detectors using the read-out chain based on the preamplifiers MPR-16 [7]. Typical energy spectra measured at the front and rear side of the X3 detector are shown in Figure 1.

The peaks are well separated, but finer structure of the peaks cannot be resolved on either the front or the rear side.
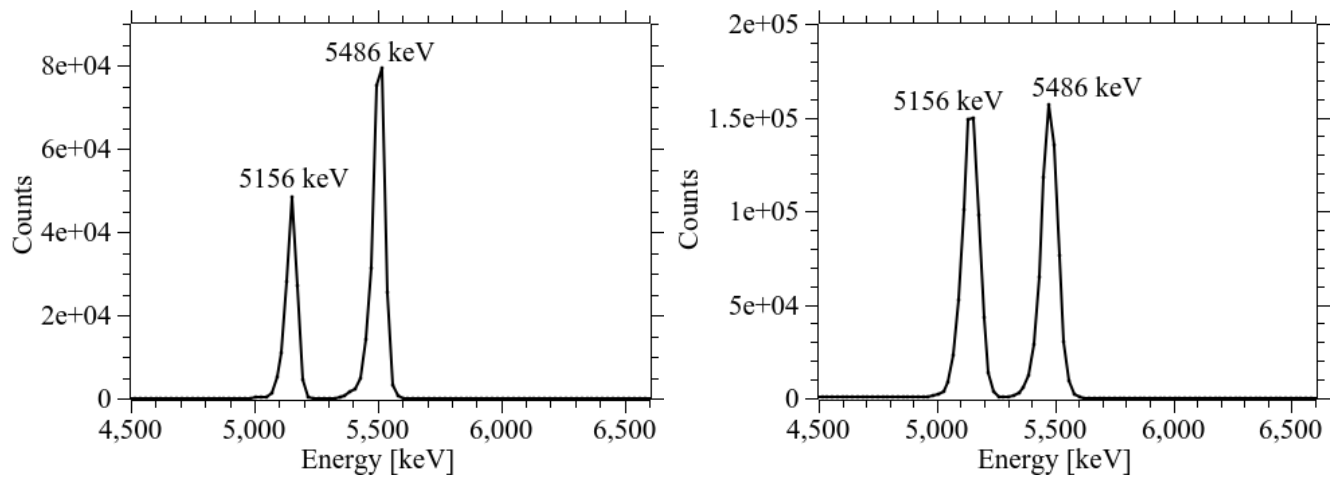

Figure 1. Energy spectra on the front and on the rear side of the X3 detector ( $\mathrm{SN}: 3199-14)$ measured with an ${ }^{241} \mathrm{Am} /{ }^{239} \mathrm{Pu} \alpha$-source using read-out chain with preamplifiers MPR-16.

The minimum and maximum energy resolution (FWHM) measured on the front side is of $37 \mathrm{keV}-$ $57 \mathrm{keV}$, depending on the specific detector. This is better than the previously published results of about $60 \mathrm{keV}$ with an $\alpha$-source for similar arrays using X3-type detectors and read-out chain with preamplifiers $[6,8]$. The results obtained on the rear side are worse, i.e. they are between $63 \mathrm{keV}-88$ $\mathrm{keV}$ in our tests and $54 \mathrm{keV}-81 \mathrm{keV}$ in the data reported by Micron Semiconductor Ltd. with ${ }^{241} \mathrm{Am}$ $\alpha$-source. The search for improving the resolution on the rear side of the X3 detectors continues, one solution is more segmentation. The best X3 detector was tested with multiplexers MUX-16. Measurements using the read-out chain with multiplexers [7] found an energy resolution which is about $50 \%$ worse than the preamplifier chain.

The position resolution of the $\mathrm{X} 3$ detector was also investigated. A mask of $3 \mathrm{~mm}$ thick was placed at a distance of $5 \mathrm{~mm}$ from the front side of the detector. Slits $1 \mathrm{~mm}$ wide are located $1 \mathrm{~mm}$ apart. The $\alpha$-source was set at $100 \mathrm{~mm}$ from the X3 detector to illuminate its entire front surface. The Figures 2 show the results of the measurement. The measured position resolution using the $\alpha$-source confirms the values published for the X3-type detectors $[8,9]$ and is about $1.5 \mathrm{~mm}$. The observed position resolution was not an intrinsic resolution, since the source had a finite size, and there was a distance between the mask and the $\mathrm{X} 3$ detector. According to our estimates, the intrinsic resolution of the $\mathrm{X} 3$ detector is less than $1 \mathrm{~mm}$.

Monitoring leakage currents during measurements showed them to be higher than specified by the manufacturer. In several cases the X3 detector displayed high leakage currents (above $700 \mathrm{nA}$ ) even at 

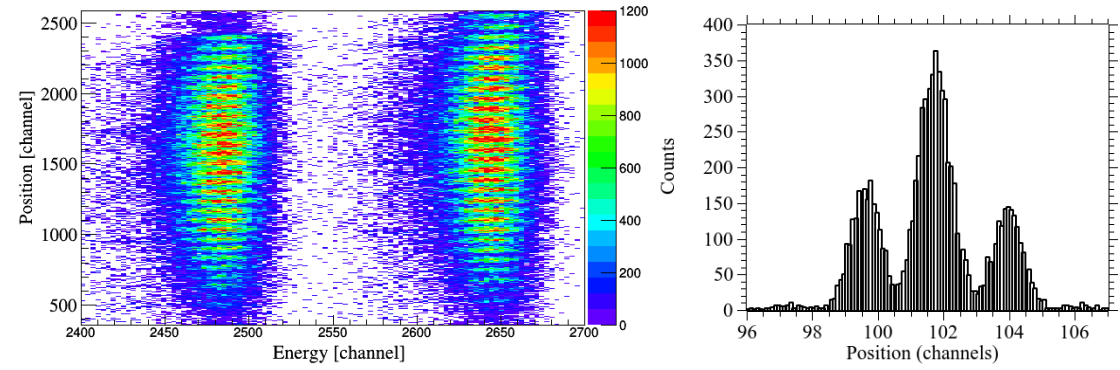

Figure 2. Energy vs position. Narrow slits of $1 \mathrm{~mm}$ are separated (on the left side). Calculation of the position resolution in the case with three illuminated slits: taking the average value of FWHM for the peaks 1, 3 and dividing it by the number of channels/mm (on the right side).

lower voltages. We also found an increase of leakage currents by $30-50 \%$ with the room temperature rises by $3-4^{\circ} \mathrm{C}$.

The ballistic deficit manifested in the dependence of pulse amplitude of the amplifier output on the charge collection time in the detector was investigated at four shaping times of $0.25,0.5,1.0$ and $2 \mu \mathrm{s}$. The effect decreased with increasing shaping time to $1 \mu \mathrm{s}$, but it again increased at $2 \mu \mathrm{s}$ due to a decrease in the 'signal-to-noise' ratio. The same behavior was found for energy resolutions. They worsened by 20-30 \% at the shortest and longest shaping times. The effect is most pronounced at the shaping time of $0.25 \mu$ s and is $30 \mathrm{keV}$.

\section{Conclusion}

At ELI-NP, a batch of forty silicon strip detectors X3 was tested, and the results were presented at the NPA8 conference. Measurement of the energy resolution on the front side showed better results than those published for similar arrays using X3 type detectors and read-out chain with preamplifiers. The leakage currents are $50 \%$ higher, depending on the detector X3 than the values measured by the manufacturer, but no correlation was found between their magnitude and energy resolution. Two readout chains were compared: the MUX-16 multiplexers and the MPR-16 preamplefiers. The read-out chain with preamplifiers is selected for the ELISSA project, because the energy resolutions measured with the MUX-16 multiplexers are $50 \%$ worse.

\section{References}

[1] O. Tesileanu et al., Rom. Rep. in Phys., 68, 699 (2016).

[2] C. Matei et al., Il Nuovo Cimento C, 39, 360 (2016).

[3] M. La Cognata et al., JINST, 12 C03079, (2017).

[4] Micron Semiconductor Ltd, Silicon detector catalogue http://www.micronsemiconductor.co.uk/silicondetector-catalogue/.

[5] S. D. Pain et al., Nucl. Instr. Meth. Phys. Res. B, 261, 1122 (2007).

[6] M. Matos et al., PoS (NIC XI), 226 (2010).

[7] Mesytec GmbH \& Co. KG - Detector Readout Systems, http://www.mesytec.com/.

[8] L. E. Pratt, PhD Thesis, LSU, (2014).

[9] E. Koshchiy, G.V. Rogachev et al., Nucl. Instr. Meth. Phys. Res A, 870, (2017). 
